\title{
COMPORTAMENTO MECÂNICO DO SOLO SOB TRÁFEGO EM DOIS SISTEMAS DE PREPARO DO SOLO
}

\author{
ANTONINO M. TERMINIELLO ${ }^{1}$, ROBERTO H. BALBUENA ${ }^{1}$, LAURA M. DRAGHI ${ }^{1}$, \\ JORGE A. CLAVERIE ${ }^{1}$, TELMO C. PALANCAR ${ }^{1}$, DANIEL JORAJURÍA ${ }^{1}$
}

\begin{abstract}
RESUMO: Os solos submetidos a sistemas de produção que não incluem o preparo do solo, estão condicionados, em sua sustentabilidade, a um manejo que priorize o acompanhamento da compactação que o tráfego de máquinas provoca. Foram realizados ensaios de campo com o objetivo de avaliar a reologia de um solo Typic Hapludert, submetido ao tráfego de veículos em dois sistemas de preparo do solo (preparo convencional - LC, e semeadura direta - SD). Os subtratamentos foram quatro intensidades de tráfego $\left(0 ; 80 ; 160\right.$ e $\left.240 \mathrm{Mg} \mathrm{km} \mathrm{ha}^{-1}\right)$. As variáveis experimentais dependentes foram: índice de cone (IC) e densidade aparente a seco (DA). Para 160 e $240 \mathrm{Mg} \mathrm{km} \mathrm{ha}^{-1}$, encontraram-se diferenças estatísticas significativas no IC em relação à testemunha. Para o LC, com as maiores intensidades de tráfego, encontraram-se valores de DA superiores a $1,83 \mathrm{Mg} \mathrm{m}^{-3}$. O comportamento mecânico do solo sob tráfego é mais diferente em SD que em LC. Existe uma relação direta entre o número de passadas e a profundidade onde se localiza a camada de maior resistência à penetração nos solos sob tratamento com LC.
\end{abstract}

PALAVRAS-CHAVE: compactação induzida, intensidade de tráfego, semeadura direta.

\section{TRAFICKED SOIL MECHANICAL BEHAVIOUR IN TWO TILLAGE SYSTEMS}

SUMMARY: A former concern related to agricultural soil, under no tillage systems, might be the state of compaction due to farm machinary traffic. Two treatments corresponding to two different tillage systems (disk plowed, LC, and no tillage, SD) included four traffic subtreatmets $(0 ; 80 ; 160$, and $240 \mathrm{Mg} \mathrm{km} \mathrm{ha}{ }^{-1}$ ) on a Typic Hapludert. Cone index (IC) and dry bulk density (DA) were the experimental dependent variables asessed. Soil reology under traffic had a different behaviour in both treatments. In both treatments for the higher traffic intensities (160 and $240 \mathrm{Mg} \mathrm{km} \mathrm{ha}^{-1}$ ) statistical significant difference $(\mathrm{P}<0,05)$ were measured when compared with the control plots, when the IC was considered. Considering LC treatment and the higher traffic intensities, the dry bulk densities values measured arised to $1.83 \mathrm{Mg} \mathrm{m}^{-3}$, the disk plowed, and no tillage under traffic soils reologies are different. If the depth of the maximum response in terms of IC measurements is considered, a direct relationship between traffic intensities and depth, could be stablished.

KEYWORDS: soil compaction, agricultural traffic, no tillage system.

\footnotetext{
${ }^{1}$ Docentes-investigadores, Depto. de Ingeniería Rural, Facultad de Ciencias Agrarias y Forestales, UNLP, Calle 60 y 119, CC31, CP. 1900, La Plata, Pcia. de Buenos Aires, Argentina, e-mail: amtermi@ceres.agro.unlp.edu.ar

Recebido pelo Conselho Editorial em: 10-1-2002

Aprovado pelo Conselho Editorial em: 14-11-2003 


\section{INTRODUCCIÓN}

La superficie sembrada en Argentina bajo el sistema de siembra directa representa aproximadamente 10 millones de hectáreas para la campaña 2000-2001, con una estimación de crecimiento a razón del 10\% anual (BRAGACHINI et al., 2000). El uso de esta tecnología puede traer aparejado problemas de sobrecompactación del suelo no solamente causado por el tránsito de máquinas agrícolas sino también por el pisoteo generado por el ganado cuando se lo incorpora dentro del sistema productivo.

En tal sentido, se estableció que en suelos arcillosos son mayores los riesgos de compactar el subsuelo a niveles que limiten la producción agrícola como mayor será también la persistencia del daño realizado. (UPADHYAYA et al., 1994).

Mientras que HÅKANSON et al. (1987) reportaron incrementos de la compactación por debajo de los $400 \mathrm{~mm}$ debidas al tránsito con cargas superiores a $60 \mathrm{kN}$ por eje, VOORHEES et al., (1978) citan un aumento de valores de resistencia a la penetración hasta una profundidad de $300 \mathrm{~mm}$ causados por el tránsito con cargas menores o iguales a $44 \mathrm{kN}$ por eje. JORAJURÍA et al., (1995), en cambio, afirman que el pasaje repetido de un tractor liviano puede inducir, por debajo del horizonte de labor, la misma compactación que un tractor más pesado con un menor número de pasadas.

SWAN et al. (1987) mostraron aumentos en densidad aparente por debajo de la profundidad de labor de herramientas de disco o reja, a la vez que consideraron que el paso de la rueda por dentro del surco compacta el suelo por debajo de la profundidad de labor habitual.

THREADGILL (1982) determinó que valores de resistencia a la penetración superiores a los 1,5 $\mathrm{MPa}$, pueden comprometer el desarrollo radicular mientras que valores superiores al rango de 2,1 a 2,5 MPa pueden impedir o detener el crecimiento de las raíces.

La densidad aparente es un parámetro que permite respaldar los datos de resistencia a la penetración con el fin de desvincular la influencia de la humedad en contenidos hídricos cercanos a capacidad de campo. Cuando esta se acerca a esos valores, el aumento de la humedad volumétrica por desaparición de macroporos luego del tráfico, hace ingresar al suelo en fase de lubricación y por tanto se miden menores valores de resistencia a la penetración, a pesar de tener un incremento de la compactación (JORAJURÍA et al., 1997). DADDOW Y WARRINGTON (1983), en estudios sobre suelos finos determinaron como umbral de crecimiento radicular a densidades aparentes de $1,65 \mathrm{Mg} \mathrm{m}^{-3}$. VEHIMEYER et al. (1948) citaron valores de $1,46 \mathrm{Mg} \mathrm{m}^{-3}$ como limitadores de dicho crecimiento.

El objetivo de este trabajo es el estudio de la variación de la reología del suelo bajo dos sistemas de labranza, cuando es sometido a tránsito.

Se trabajó sobre la hipótesis de que la compactación inducida por el tránsito se manifiesta de igual manera en profundidad independientemente del sistema de labranza.

\section{MATERIALES Y MÉTODOS}

\section{Del suelo y su identificación}

Los ensayos fueron realizados en la Estación Experimental Agropecuaria - INTA, Concepción del Uruguay, Provincia de Entre Ríos, Argentina, a los $32^{\circ} 30^{\prime}$ de LS y $58^{\circ} 20^{\prime}$ LO. El suelo del ensayo pertenece a la clasificación Typic Hapludert (SOIL TAXONOMY, 1999), de la serie Clara. Los horizontes presentes son los siguientes: $\mathrm{A}_{11}: \mathrm{de}$ 0-15 cm, negro en húmedo y gris oscuro en seco, arcilloso, de bloques subangulares muy finos a medianos, duros en seco y firmes en húmedo. $\mathrm{A}_{12}$ : 15$30 \mathrm{~cm}$, negro en húmedo y gris oscuro en seco, arcilloso, bloques subangulares medianos; duro, friable. $\mathrm{B}_{21}$ : 30-50 cm, negro en húmedo y gris oscuro en seco; arcilloso; con bloques subangulares 
medios a gruesos, moderado, duro, firme, muy plástico. $\mathrm{B}_{22}: 50-70 \mathrm{~cm}$. Pardo grisáceo muy oscuro en seco, gris oscuro a negro en húmedo; arcilloso; bloques angulares gruesos, muy duro, firme, muy plástico. $\mathrm{BC}_{\mathrm{ca}}: 70-100 \mathrm{~cm}$, pardo grisáceo en seco y gris muy oscuro en húmedo, bloques angulares gruesos, firme, muy plástico. $\mathrm{C}_{1}: 100-125 \mathrm{~cm}$, mezcla de pardo y gris oscuro en húmedo; arcillo limoso; masivo a bloques subangulares, duro, firme, plástico. $\mathrm{C}_{2}: 125-155 \mathrm{~cm}$, pardo en húmedo; arcillo limoso, masivo a bloques subangulares, duro, friable, plástico.

\section{De las variables experimentales}

Dos condiciones del suelo definen dos tratamientos: 1 - Suelo bajo labranza convencional (LC): Historia del lote: en marzo de 1999, una arada con arado de cinco discos de una masa total de 2,9 $\mathrm{Mg}$, dos pasajes de rastra de casquetes de $2,8 \mathrm{Mg}$ y un pasaje de rastra de dientes. Posteriormente el lote fue sembrado con una mezcla para praderas, recibiendo una fertilización de $80 \mathrm{~kg} \mathrm{ha}^{-1}$ de super fosfato triple. 2 - Suelo bajo siembra directa (SD). Historia del lote: se sembró en marzo de 1999 con la misma mezcla, fertilizándose con $60 \mathrm{~kg} \mathrm{ha}^{-1}$ de fosfato di amónico. El tráfico, una vez instalada la pastura fue el mismo en ambos terrenos, limitándose al tráfico animal en pastoreo directo con una carga de 50 novillos por ha durante tres días y clausura para recuperación de la pastura de 30 días. Cada tratamiento está ubicado sobre un lote de 4 ha partido en cuatro con dos repeticiones cada uno.

\section{Del tractor}

Los subtratamientos sobre las parcelas estuvieron constituidos por pasajes repetidos del tractor estableciéndose una secuencia de $5 ; 10$ y 15 pasadas, tomándose como testigo al tratamiento sin tránsito. Cada subtratamiento significó, respectivamente, una intensidad de tráfico de: $80 ; 160$ y $240 \mathrm{Mg} \mathrm{km} \mathrm{ha}{ }^{-1}$. El tractor utilizado fue un Deutz AX140, de diseño FWA (ASAE), 99,3 kW de potencia, con una masa total de 6,29 Mg, distribuida en 2,39 Mg sobre el tren delantero y 3,9 $\mathrm{Mg}$ sobre el trasero. Los rodados delanteros: 13,6 x 28 y los traseros 18,4 x 38 .

La intensidad de tránsito que recibieron los lotes se indica en la Tabla 1.

TABLA 1. Intensidad de tránsito para labranza convencional (LC) y siembra directa (SD)

\begin{tabular}{clccc}
\hline Tratamiento & Implemento & Ancho de Labor $(\mathrm{m})$ & Número de Pasadas & $\left.(\mathrm{Mg} \mathrm{km} \mathrm{ha})^{-1}\right)$ \\
\hline LC & Arado de discos & 1,80 & 1 & 29,7 \\
LC & Rastra de discos & 3,80 & 2 & 28,4 \\
LC & Vibrocultivador & 4,00 & 1 & 13,5 \\
LC & Sembradora & 3,68 & 1 & 14,7 \\
LC & Fertilizadora & 8,00 & 1 & 6,8 \\
\hline LC & Total & & 1 & 93,1 \\
\hline SD & Sembradora & 3,68 & 1 & 14,7 \\
SD & Fertilizadora & 8,00 & & 6,8 \\
\hline SD & Total & & 11,5 \\
\hline
\end{tabular}

\section{De las variables independientes}

El comportamiento del suelo, para los dos tratamientos (LC y SD) fue evaluada analizando: la resistencia a la penetración determinada con un penetrómetro de cono (ASAE S 313.2, 1992) Rimik CP20 electrónico con definición de profundidad por ultrasonido, relevándose el perfil hasta una profundidad de $575 \mathrm{~mm}$ con trece repeticiones sobre cada parcela experimental. A partir de estos datos se obtuvo el valor del índice de cono (IC) cada $50 \mathrm{~mm}$ del perfil analizado.

La densidad aparente en seco se determinó mediante sonda nuclear gammamétrica Troxler 3440, hasta los $300 \mathrm{~mm}$ de profundidad con tres repeticiones sobre cada parcela experimental a intervalos de 
0 hasta 50-100-150-200-250 y $300 \mathrm{~mm}$ de profundidad. Para el tratamiento SD no se relevaron datos para 15 pasadas. Como variable explicativa se midió la humedad gravimétrica, tomándose valores a intervalos de 0-150 mm, 150-300 $\mathrm{mm}$ y 300-600 mm, con cuatro repeticiones (Tabla 2).

TABLA 2. Humedad gravimétrica media $(\mathrm{p} / \mathrm{p})$ medida al momento de instalar el ensayo de tráfico.

\begin{tabular}{cccc}
\hline \multirow{2}{*}{ Tratamientos } & \multicolumn{3}{c}{ Profundidad (mm) } \\
\cline { 2 - 4 } & $0-150$ & $150-300$ & $300-600$ \\
\hline SD & $27,81 \mathrm{a}$ & $29,49 \mathrm{a}$ & $31,23 \mathrm{a}$ \\
LC & $29,93 \mathrm{a}$ & $30,30 \mathrm{a}$ & $33,09 \mathrm{~b}$ \\
\hline
\end{tabular}

Valores seguidos de igual letra en la fila consignan diferencias no significativas $(\mathrm{P}<0,05)$ - Test LSD.

\section{Del diseño experimental}

\section{Condición de Siembra Directa}

Para cada intervalo de profundidad en particular se analizó el efecto del tránsito sobre las variables Resistencia a la Penetración y Densidad Aparente. En relación a la primera cada ensayo simple contó con cuatro intensidades de tránsito (testigo, $80 ; 160$, y $240 \mathrm{Mg} \mathrm{km} \mathrm{ha}^{-1}$ ) con 8 repeticiones cada una. El delineamiento experimental fue enteramente al azar contando con 32 parcelas, con un dimensionamiento de $12 \mathrm{~m}$ de ancho por $100 \mathrm{~m}$ de largo. Se efectuaron 13 determinaciones de esta variable por parcela, a 11 profundidades distintas. Para la variable densidad aparente el ensayo fue similar, efectuándose 3 observaciones por parcela, a 6 intervalos de profundidad.

\section{Condición de Labranza Convencional}

Ídem Siembra Directa.

Sobre los datos de los parámetros relevados se efectuó un análisis de la varianza, utilizándose el test de LSD $(\mathrm{P} \leq 0,05)$ para diferenciar las medias.

\section{RESULTADOS Y DISCUSIÓN}

Del análisis de los valores de índice de cono (IC) (Tabla 3), para el tratamiento de LC se visualiza un incremento del parámetro para los subtratamientos de 160 y $240 \mathrm{Mg} \mathrm{km} \mathrm{ha}^{-1}$ con diferencias estadísticas significativas en relación al testigo, pero sin diferenciación entre ellos, para todo el perfil de suelo evaluado. Posiblemente el suelo presentó un estado de masificación tal que permitió el incremento IC desde la superficie conforme se aumentara el número de pasadas hasta los 250-300 mm. Esta condición de densificación del perfil se corrobora en el gráfico de densidad aparente (Figura 1) donde se dan los mayores valores en el estrato que va desde los 250 a los $300 \mathrm{~mm}$.

A partir de los 300-350 mm de profundidad, si bien continuaron manifestándose las diferencias anteriormente mencionadas en el IC, con respecto al testigo, los mayores valores absolutos se dieron para el subtratamiento de $160 \mathrm{Mg} \mathrm{km} \mathrm{ha}^{-1}$. Esto pudo deberse a que la mayor intensidad de tránsito fuese motivo de una reorganización del estado poroso del suelo al punto tal de provocar un incremento del volumen de agua y, por tanto, una disminución de los valores de la variable mencionada para la intensidad de tránsito de $240 \mathrm{Mg} \mathrm{km} \mathrm{ha}^{-1}$. Esto sería posible dado la estrecha dependencia que tiene el parámetro resistencia a la penetración con la humedad, sin que ello signifique una situación de menor compactación de ese suelo (JORAJURÍA et al., 1997). Lo anteriormente mencionado pudo ser influenciado, también, por el cambio de horizonte de A12 a B21 que se evidencia a lo $300 \mathrm{~mm}$. 
Solamente se identificaron valores de IC que comprometerían el desarrollo radicular (THREADGILL, 1982) a partir de los $350 \mathrm{~mm}$, para el subtratamiento de $160 \mathrm{Mg} \mathrm{km} \mathrm{ha}{ }^{-1}$, a partir de los $400 \mathrm{~mm}$ para el de $240 \mathrm{Mg} \mathrm{km} \mathrm{ha}^{-1} \mathrm{y}$ a partir de los $450 \mathrm{~mm}$ para el de $80 \mathrm{Mg} \mathrm{km} \mathrm{ha}^{-1}$.

TABLA 3. Valores de índice de cono para labranza convencional (MPa).

\begin{tabular}{ccccc}
\hline \multirow{2}{*}{ Profundidad $(\mathrm{mm})$} & \multicolumn{4}{c}{ Subtratamientos $\left(\mathrm{Mg} \mathrm{km} \mathrm{ha}^{-1}\right)$} \\
\cline { 2 - 5 } & Testigo & 80 & 160 & 240 \\
\hline $0-50$ & $0,92 \mathrm{a}$ & $1,06 \mathrm{~b}$ & $1,08 \mathrm{~b}$ & $1,08 \mathrm{~b}$ \\
$50-100$ & $0,90 \mathrm{a}$ & $1,04 \mathrm{~b}$ & $1,10 \mathrm{~b}$ & $1,11 \mathrm{~b}$ \\
$100-150$ & $0,95 \mathrm{a}$ & $1,03 \mathrm{ab}$ & $1,16 \mathrm{~b}$ & $1,19 \mathrm{~b}$ \\
$150-200$ & $0,93 \mathrm{a}$ & $1,10 \mathrm{~b}$ & $1,17 \mathrm{~b}$ & $1,25 \mathrm{~b}$ \\
$200-250$ & $0,93 \mathrm{a}$ & $1,20 \mathrm{~b}$ & $1,26 \mathrm{~b}$ & $1,32 \mathrm{~b}$ \\
$250-300$ & $0,99 \mathrm{a}$ & $1,31 \mathrm{~b}$ & $1,36 \mathrm{~b}$ & $1,36 \mathrm{~b}$ \\
$300-350$ & $1,07 \mathrm{a}$ & $1,38 \mathrm{~b}$ & $1,47 \mathrm{~b}$ & $1,42 \mathrm{~b}$ \\
$350-400$ & $1,12 \mathrm{a}$ & $1,43 \mathrm{ab}$ & $1,57 \mathrm{~b}$ & $1,48 \mathrm{~b}$ \\
$400-450$ & $1,18 \mathrm{a}$ & $1,47 \mathrm{ab}$ & $1,67 \mathrm{~b}$ & $1,59 \mathrm{~b}$ \\
$450-500$ & $1,19 \mathrm{a}$ & $1,54 \mathrm{ab}$ & $1,78 \mathrm{~b}$ & $1,65 \mathrm{~b}$ \\
$500-550$ & $1,21 \mathrm{a}$ & $1,58 \mathrm{ab}$ & $1,87 \mathrm{~b}$ & $1,75 \mathrm{~b}$ \\
\hline
\end{tabular}

Valores seguidos de igual letra en la fila consignan diferencias no significativas $(\mathrm{P}<0,05)$ - Test LSD

Del análisis de los datos de IC para SD (Tabla 4) se evidencia que, al igual que para LC, las mayores intensidades de tránsito presentaron diferencias estadísticas significativas para todo el perfil en relación al testigo. Sin embargo para LC las diferencias con respecto al testigo fueron algo mayores, con lo que se puede suponer que el suelo bajo este sistema de labranza se mostró mas sensible a los tratamientos de tránsito. Posiblemente el contenido de humedad del suelo posibilitó que se incrementara el IC en la medida que se aumentara el tránsito sobre la misma senda .

Para el estrato de 0-50 se encontraron diferencias estadísticas significativas en todas las intensidades de tránsito con respecto al testigo. Esto pudo deberse a la mayor densificación del perfil visualizada en el horizonte de $50 \mathrm{~mm}$ (Figura 2) se constituyó en una capa soporte que permitió la diferenciación anteriormente mencionada para cualquier número de pasadas.

A partir del estrato de 100-150 y hasta el final del perfil se obtuvieron valores de IC superiores a los 1,5 MPa para el subtratamiento de mayor intensidad de tránsito que comprometerían el desarrollo radicular (THREADGILL, 1982), lo mismo ocurrió para el subtratamiento de $160 \mathrm{Mg} \mathrm{km} \mathrm{ha}{ }^{-1}$ a partir del estrato de los 200-250 mm. Lo anteriormente mencionado pudo motivarse por el estado de humedad que presentaba el suelo que posibilitó la reacción del mismo al tránsito para los mayores números de pasadas, con respecto al testigo, a pesar que el tractor utilizado no superaba los $60 \mathrm{Mg}$ por eje citados por HAKÅNSON et al., (1987) como causantes de compactación en horizontes subsuperficiales. A diferencia de lo enunciado por VOORHEES et al. (1978) tractores con cargas por eje menores a $44 \mathrm{Mg}$ también produjeron compactación subsuperficial. Se evidencia, además, que pasajes sucesivos de un tractor liviano inducen a la compactación subsuperficial, esto concuerda con lo manifestado por JORAJURÍA et al. (1995) manifestándose tanto para e SD como para LC.

Del análisis de la densidad aparente, tanto para LC como para SD (Figuras 1 y 2 ), se evidencia una densificación del perfil en el estrato de $50 \mathrm{~mm}$. Esto podría deberse al pisoteo del ganado que provocó una disminución tal de la porosidad del suelo a esa profundidad que no permitió la manifestación de los subtratamientos de tránsito realizados para la condición de SD y, solamente, para la mayor intensidad de tránsito para el tratamiento LC. Los valores encontrados de este parámetro, para el horizonte mencionado en SD, fueron superiores a los 1,46 $\mathrm{Mg} \mathrm{m}^{-3}$ citados por VEHIMEYER et 
al. (1948) como limitante del crecimiento de las raíces. Por debajo de dicho estrato no se manifestaron limitaciones en el citado parámetro para el crecimiento radicular. La existencia de un horizonte más denso a los $50 \mathrm{~mm}$ se pudo haber comportado como capa soporte que permitió que se manifestaran valores mayores en superficie en comparación a los obtenidos a partir de los $100 \mathrm{~mm}$. Para LC, la situación hacia la superficie y por debajo del horizonte de $50 \mathrm{~mm}$ es similar que para el tratamiento SD aunque, en el estrato de $50 \mathrm{~mm}$, los valores encontrados superarían el umbral de $1,46 \mathrm{Mg} \mathrm{m}^{-3}$ anteriormente mencionado solamente para los subtratamientos de mayor intensidad de tránsito.

TABLA 4. IC en Siembra Directa (MPa).

\begin{tabular}{ccccc}
\hline \multirow{2}{*}{ Profundidad $(\mathrm{mm})$} & \multicolumn{4}{c}{ Subtratamientos $\left(\mathrm{Mg} \mathrm{km} \mathrm{ha}^{-1}\right)$} \\
\cline { 2 - 5 } & Testigo & 80 & 160 & 240 \\
\hline $0-50$ & $0,93 \mathrm{a}$ & $1,08 \mathrm{~b}$ & $1,14 \mathrm{~b}$ & $1,27 \mathrm{c}$ \\
$50-100$ & $0,97 \mathrm{a}$ & $1,04 \mathrm{~b}$ & $1,22 \mathrm{~b}$ & $1,35 \mathrm{~b}$ \\
$100-150$ & $1,06 \mathrm{a}$ & $1,12 \mathrm{a}$ & $1,36 \mathrm{~b}$ & $1,51 \mathrm{~b}$ \\
$150-200$ & $1,20 \mathrm{a}$ & $1,23 \mathrm{a}$ & $1,48 \mathrm{~b}$ & $1,64 \mathrm{~b}$ \\
$200-250$ & $1,32 \mathrm{a}$ & $1,39 \mathrm{a}$ & $1,67 \mathrm{~b}$ & $1,83 \mathrm{~b}$ \\
$250-300$ & $1,46 \mathrm{a}$ & $1,53 \mathrm{a}$ & $1,86 \mathrm{~b}$ & $2,01 \mathrm{~b}$ \\
$300-350$ & $1,55 \mathrm{a}$ & $1,74 \mathrm{a}$ & $2,07 \mathrm{~b}$ & $2,13 \mathrm{~b}$ \\
$350-400$ & $1,62 \mathrm{a}$ & $1,98 \mathrm{~b}$ & $2,36 \mathrm{c}$ & $2,29 \mathrm{c}$ \\
$400-450$ & $1,80 \mathrm{a}$ & $2,13 \mathrm{a}$ & $2,62 \mathrm{~b}$ & $2,46 \mathrm{~b}$ \\
$450-500$ & $2,02 \mathrm{a}$ & $2,56 \mathrm{~b}$ & $2,58 \mathrm{~b}$ & $2,89 \mathrm{~b}$ \\
$500-550$ & $2,26 \mathrm{a}$ & $2,93 \mathrm{bc}$ & $3,33 \mathrm{c}$ & $2,72 \mathrm{~b}$ \\
\hline
\end{tabular}

Valores seguidos de igual letra en la fila consignan diferencias no significativas $(\mathrm{P}<0,05)$ del Test LSD.

Al observar la Figura 1, se comprueba un incremento en los valores entre los 250 y los $300 \mathrm{~mm}$. Lo anteriormente mencionado pudo deberse a la coexistencia de distintas circunstancias, por un lado, a un cambio de horizonte pedogenético ya que a partir de los $300 \mathrm{~mm}$ y hasta los $500 \mathrm{~mm}$ aparece un B21 y, por el otro, a que inmediatamente por encima de esa profundidad se localizaría un piso de labor que según SWAN et al. (1987) sería causante de un aumento de la densidad aparente inmediatamente por debajo del mismo. Ambas situaciones, junto con una posible lixiviación de arcillas, pudieron conjugarse y dar como resultado la masificación del suelo encontrada a los $300 \mathrm{~mm}$ de profundidad. Los valores hallados en este estrato superaron los $1,65 \mathrm{Mg} \mathrm{m}^{-3}$ citados por DADDOW \& WARRINGTON (1983) como umbral de crecimiento vegetal.

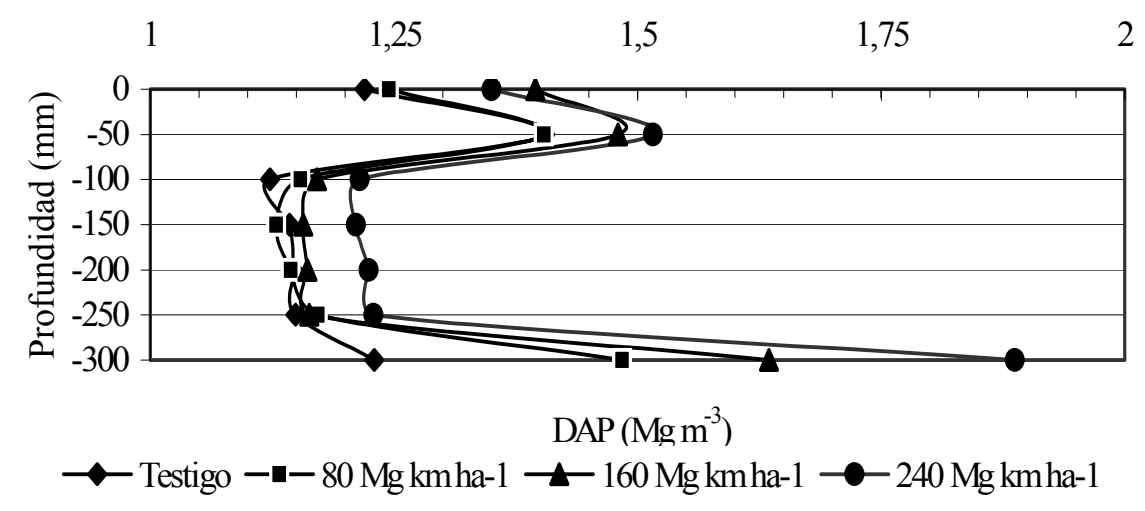

FIGURA 1. Densidad aparente para labranza convencional $\left(\mathrm{Mg} \mathrm{m}^{-3}\right)$. 


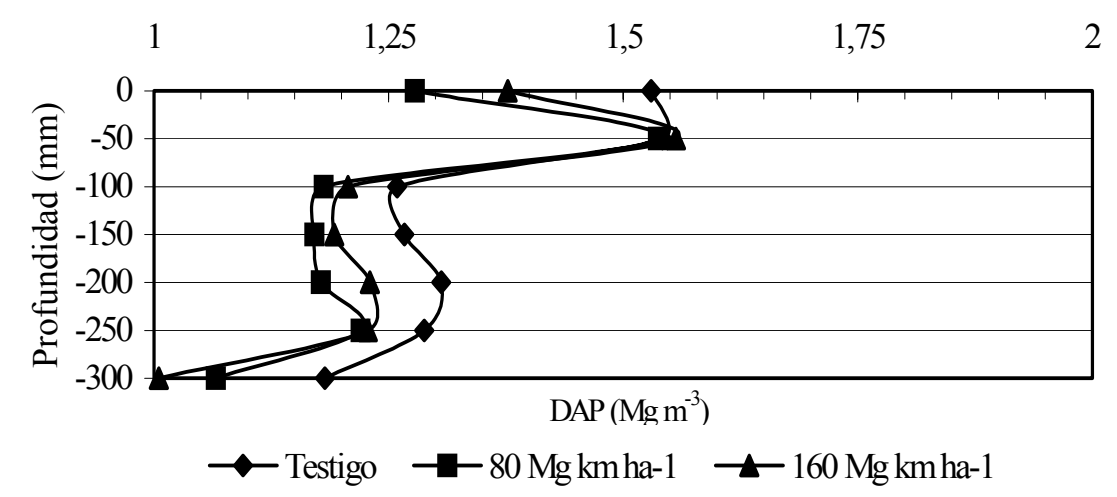

FIGURA 2. Densidad aparente para siembra directa $\left(\mathrm{Mg} \mathrm{m}^{-3}\right)$.

Del análisis de los datos de densidad aparente después del tránsito, (Figuras 1 y 2), se evidencia un comportamiento mecánico similar en los dos tratamientos hasta el estrato de $250 \mathrm{~mm}$. Sin embargo, a partir de esa profundidad, la reología es claramente opuesta atendiendo a una condición inicial también diferente entre tratamientos. Esto es que antes de aplicados los subtratamientos de tránsito, la condición inicial del suelo bajo SD mostraba ya una condición de menor densidad aparente en el horizonte de $300 \mathrm{~mm}$ respecto del de $250 \mathrm{~mm}$. Luego del tránsito la densidad aparente medida a $300 \mathrm{~mm}$ fue inferior a la condición inicial. No obstante esta aparente disminución de la densidad no puede ser corroborada por los datos de IC, dado que con cada subtratamiento de tráfico se registraron incrementos de IC medidos inmediatamente después del tránsito y comparados a la situación anterior testigo. Este dato contradictorio, observable en el suelo bajo SD, puede explicarse, en principio, por una mayor dificultad de preparación de la superficie donde se apoya la sonda gammamétrica, en la zona de la impronta del rodado. La presencia de una masa radicular importante y el rastrojo remanente crearon dificultades al momento de hacer el plano del suelo donde se apoya la sonda en la huella. De esta manera se generaron espacios entre el suelo y la base de la sonda que ésta integra al momento de medir en el perfil que irradia, disminuyendo, entonces, el dato de densidad aparente debajo de la huella que dejaron las tres intensidades de tráfico aplicadas.

Las diferencias medidas en la humedad presente en esa profundidad entre ambos tratamientos, fue del orden de 1 a $3 \%$ mayores para el suelo bajo LC. Sobre tan escasa diferencia de humedad gravimétrica debe desecharse una hipótesis de diferencia en IC debida a variaciones en la humedad volumétrica. Para el tratamiento considerado, el subtratamiento de $80 \mathrm{Mg} \mathrm{km} \mathrm{ha}^{-1}$ provocó el mayor incremento del IC en el rango 250 - $300 \mathrm{~mm}$ de profundidad. Por otro lado, la segunda intensidad de tránsito (160 Mg km ha $\left.{ }^{-1}\right)$ hizo al suelo reaccionar con un incremento máximo del IC en el rango de 500 a $550 \mathrm{~mm}$ de profundidad. El suelo sometido a la máxima intensidad de tráfico, siguió el mismo patrón de comportamiento, maximizando su reacción a la profundidad de 500 a $550 \mathrm{~mm}$.

En la Tabla 5 se puede apreciar los incrementos porcentuales de IC para los subtratamientos de tránsito con respecto al testigo. Puede observarse que el suelo bajo SD mostró un comportamiento diferente. La menor intensidad de tráfico y considerando sólo desde la superficie y hasta los $300 \mathrm{~mm}$. de profundidad, provocó incrementos medios de sólo 7\% en el IC, mientras que a partir de allí y hasta la profundidad máxima evaluada el incremento promedió $22 \%$. Un comportamiento similar tuvo el suelo luego de la intensidad de tráfico intermedia $\left(160 \mathrm{Mg} \mathrm{km} \mathrm{ha}^{-1}\right)$ ya que el primer intervalo considerado ( 0 a $300 \mathrm{~mm}$ ) el incremento de IC promedió el $26 \%$, en cambio en el rango más profundo (300 a $550 \mathrm{~mm}$.) estos incrementos arrojaron una media de 55\%. Sin embargo, al considerar el suelo bajo el subtratamiento $240 \mathrm{Mg} \mathrm{km} \mathrm{ha}{ }^{-1}$, siempre en estos dos rangos de profundidades, el resultado tiende a igualarse en ambos rangos, e inclusive reaccionando con incrementos algo mayores el rango más superficial, a $300 \mathrm{~mm}$. (38\%) respecto al 36\% promediado de 300 a $550 \mathrm{~mm}$. 
TABLA 5. Incrementos porcentuales de índice de cono con respecto al testigo.

\begin{tabular}{|c|c|c|c|c|c|c|}
\hline \multirow{3}{*}{ Profundidad (mm) } & \multicolumn{6}{|c|}{ Subtratamientos $\left(\mathrm{Mg} \mathrm{km} \mathrm{ha}^{-1}\right)$} \\
\hline & \multicolumn{2}{|c|}{80} & \multicolumn{2}{|c|}{160} & \multicolumn{2}{|c|}{240} \\
\hline & $\mathrm{LC}$ & SD & $\mathrm{LC}$ & SD & $\mathrm{LC}$ & SD \\
\hline $0-50$ & 15,2 & 16,1 & 17,4 & 22,6 & 17,4 & 36,5 \\
\hline $50-100$ & 15,5 & 7,2 & 22,2 & 25,7 & 23,3 & 39,2 \\
\hline $100-150$ & 8,4 & 5,7 & 22,1 & 28,3 & 25,3 & 42,5 \\
\hline $150-200$ & 18,3 & 2,5 & 25,8 & 23,3 & 34,4 & 36,6 \\
\hline $200-250$ & 29,0 & 5,3 & 35,5 & 26,5 & 41,9 & 38,6 \\
\hline $250-300$ & 32,3 & 4,8 & 37,4 & 27,4 & 37,4 & 37,7 \\
\hline $300-350$ & 28,9 & 12,2 & 37,4 & 33,5 & 32,7 & 37,4 \\
\hline $350-400$ & 27,7 & 22,2 & 40,2 & 45,7 & 32,1 & 41,3 \\
\hline $400-450$ & 24,6 & 18,3 & 41,5 & 45,5 & 34,7 & 36,7 \\
\hline $450-500$ & 29,4 & 26,7 & 49,6 & 27,7 & 38,6 & 43,1 \\
\hline $500-550$ & 30,6 & 29,6 & 54,5 & 47,3 & 44,6 & 20,3 \\
\hline
\end{tabular}

LC - labranza convencional; SD - siembra directa

A modo de balance de la variable experimental índice de cono se puede considerar respecto al suelo bajo LC que las mayores intensidades de tránsito hicieron reaccionar al suelo con picos de IC a profundidades mayores. Prácticamente no hay diferencias en la media de los incrementos en el IC provocados por los subtratamientos 160 y $240 \mathrm{Mg} \mathrm{km} \mathrm{ha}^{-1}$, inclusive el de menor intensidad de tráfico provocó incrementos levemente superiores (34,8\% vs. 32,9\%). No obstante esto, los valores relevados de densidad aparente sí evidencian una mayor compactación inducida por el subtratamiento de $240 \mathrm{Mg} \mathrm{km} \mathrm{ha}{ }^{-1}$ (Fig. 1). La hipótesis para explicar este contradictorio resultado puede esgrimirse considerando la variable explicativa humedad gravimétrica presente, algo mayor para esta condición de suelo (31\%), cercana ya a la capacidad de campo.

Tomando en cuenta lo establecido para situaciones similares por JORAJURÍA et al. (1997), el descenso en los valores del IC podría deberse a un incremento en la humedad volumétrica del perfil, inducido por la compactación dada por el tráfico y puesta en evidencia por el incremento de la DA.

En lo que respecta al suelo bajo SD, el mismo manifestó una reología diferente. Para facilitar el análisis y considerando dos rangos de profundidades, (superior 0-300 mm, e inferior 300-550 $\mathrm{mm}$ ), puede verse que en las dos primeras intensidades de tráfico si bien se mantiene un incremento mayor del IC para el rango inferior de profundidad, los mismos fueron menores. Para el caso de la máxima intensidad de tráfico $\left(240 \mathrm{Mg} \mathrm{km} \mathrm{ha}{ }^{-1}\right)$ los incrementos de IC, para los rangos de profundidad evaluados, fueron prácticamente iguales. Por lo tanto en el suelo bajo el tratamiento SD no se cumple la citada relación directamente número de pasadas y profundidad de la localización de la zona de mayor incremento de IC. El suelo pareciera reaccionar de una manera más homogénea que el suelo bajo tratamiento con laboreo convencional. No aparecen aquí reacciones tan diferentes a los mismos subtratamientos de tráfico y, en todo caso, las diferencias cuando las hubo, fueron menos significativas entre rangos de profundidades superior e inferior.

Por otro lado, para la intensidad de tráfico mayor $\left(240 \mathrm{Mg} \mathrm{km} \mathrm{ha}^{-1}\right)$, los resultados evidencian, con bastante claridad, que los incrementos en el IC han sido mayores en el suelo bajo este tratamiento. Esto estaría indicando una reacción diferente del suelo estrechamente relacionada a la intensidad de tráfico puesto que para el subtratamiento de $80 \mathrm{Mg} \mathrm{km} \mathrm{ha}^{-1}$ se registraron mayoritariamente menores incrementos del parámetro mencionado, mostrando el segundo subtratamiento $\left(160 \mathrm{Mg} \mathrm{km} \mathrm{ha}{ }^{-1}\right)$ una situación intermedia, donde a algunas profundidades se registraron valores mayores que para LC, mientras que para otras profundidades los incrementos estuvieron por debajo. 


\section{CONCLUSIONES}

El comportamiento mecánico del suelo bajo tráfico es diferente en siembra directa que en laboreo convencional

Existe una relación directa entre la intensidad de tránsito y la profundidad adonde se localiza la capa de mayor resistencia a la penetración en los suelos bajo tratamiento de laboreo convencional.

La variabilidad de la reacción del suelo bajo siembra directa responde más a la intensidad de tránsito que al rango de profundidad considerado.

\section{REFERÊNCIAS BIBLIOGRÁFICAS}

AMERICAN SOCIETY OF AGRICULTURAL ENGINEERS (ASAE) - Standard of soil cone penetrometer. St Joseph, 1992 p.611 (ASAE S 313.2)

BRAGACHINI, M.; MÉNDEZ A.; VON MARTÍN, A.; MONCHAMP, J. Eslabonamiento productivo del sector de maquinaria agrícola Argentina. Buenos Aires: Consejo Federal de Inversiones, 2000. $104 \mathrm{p}$.

DADDOW, R.L.; WARRINGTON, G.E. Growth-limiting soil bulk densities as influenced by soil texture. Fort Collins: USDA, 1983. 162 p.

HAKÅNSON, Y.; VOORHES W.V.; ELONEN, P.; RAGHAVAN, G.S.V.; LOWERY, B.; WIJK, A.L.M. van; RASMUSSEN, K.; RILEY, H. Effect of high axle-load traffic on subsoil compaction and crop yield in humid regions with annual freezing. Soil and Tillage Research, Amsterdam, v.10, p.25968, 1987.

JORAJURIA, D.; DRAGHI, L.; ARAGON, A. Compactación del suelo bajo tráfico repetido. Investigación Agraria: Producción y Protección Vegetales, Madrid, v.10, n.3, p.473-83 , 1995.

JORAJURIA, D.; DRAGHI, L.; ARAGON, A. The effect of vehicle weight on the distribution of compaction with depth and the yield of a Lolium/Trifolium grassland. Soil \& Tillage Research, Amsterdam, v.4, p.1-12, 1997.

SWAN, J.; MONCRIEF, J.; VOORHEES, W. Soil compaction. Causes, effects, and control. USA: Minnesota Extension Service, University of Minnesota, 1987. (AG-BU-3115)

SOIL TAXONOMY. A basic system of soil classification for making and interpreting soil surveys. 2. ed. Soil Survey Staff, 1999. (n.436)

THREADGILL, E.D. Residual tillage effects as determined by cone index. Transaction of the ASAE, St. Joseph, v.25, n.4, p.859-963, 1982.

UPADHYAYA, S.K.; CHANCELLOR, W.; PRERUMPRAL, J.; SCHAFER, R.S.; GILL, W.; VANDERBERG E. Advances in Soil Dynamics. St. Joseph: De Vore-Hansen, 1994. 313 p.

VEIHMEYER, F.J.; HENDRICKSON A.H. Soil density and root penetration. Soil Science, Baltimore, v.65, p.487-93, 1948.

VOORHESS, W.B.; SENST, C.G.; NELSON, W.W. Compaction and soil structure modification by wheel traffic in the northern Corn Belt. Soil Science Society of America Journal, Madison, v.50, p.72833, 1978. 